\title{
Zacatecas: violencia, inseguridad y crimen organizado en el siglo XXI Ramiro Villasana Villalobos*
}

\author{
La vinculación entre el crimen organizado y la violencia e inseguridad existentes \\ en Zacatecas ha constituido un fenómeno con repercusiones para la población \\ de todas las edades. Los actores involucrados en el desarrollo y combate del conflicto \\ son diversos. Asimismo, durante el presente siglo, la entidad zacatecana ha manifestado se- \\ mejanzas y divergencias respecto a lo acaecido con las problemáticas de seguridad \\ en otros territorios nacionales, siendo un caso representativo la cooperación \\ de autoridades públicas con agrupaciones delictivas.
}

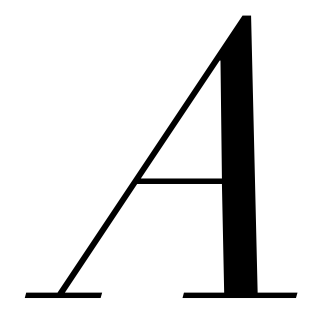

CTUALMENTE, ENTRE los instrumentos jurídicos existentes para combatir la violencia en Zacatecas, caben destacar la Ley para Prevenir y Atender la Violencia Familiar (2003) y la Ley de Acceso de las Mujeres a una Vida Libre de Violencia (2009). Un par de normas desarrolladas con el objetivo de brindar seguridad social a dos agrupaciones de suma importancia en el estado: la familia y las mujeres. ${ }^{1}$

No obstante, la violencia no se restringe al ámbito familiar o de género. Hoy en día es fundamental la

* Estudiante de la Maestría en Relaciones Económicas Internacionales y Cooperación en la Universidad de Guadalajara, México. instauración de nuevas estrategias para el combate a los diferentes tipos de violencia suscitados en los territorios estatales.

Sin duda, una de las problemáticas sociales que han atentado en gran medida contra la seguridad en el siglo XXI es el narcotráfico. Derivado de esa actividad ilícita, en Zacatecas, como en todo México, ha tenido lugar el fallecimiento y secuestro de una vasta cantidad de personas. ${ }^{2}$

Al iniciar el presente siglo, entre los delitos federales, el narcotráfico constituyó el rubro con más delincuentes sentenciados en Zacatecas y se asocia con una problemática nacional de alto impacto en niños y adolescentes, ya que en 20II, en el país, más de 25,000 menores de edad estaban vinculados al crimen organizado, 

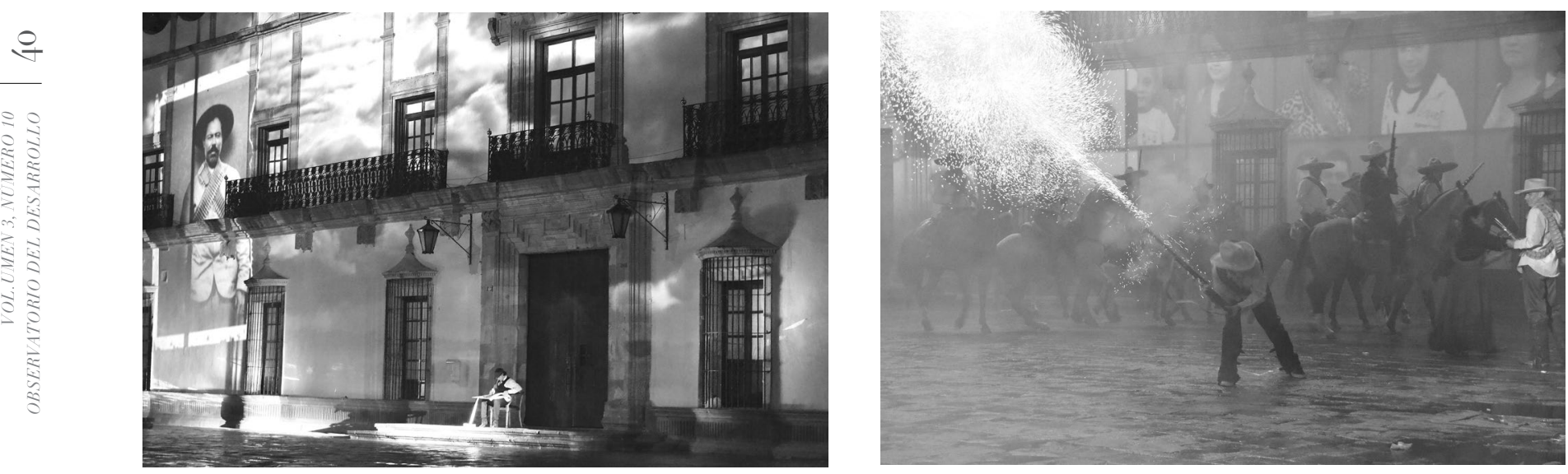

El gobierno local destino 450 millones de pesos para realizar los festejos de la Toma de Zacatecas en actividades espectaculares que no dejaron grandes enseñanzas ni beneficios tangibles para la población.

Foto: Jorge VázQuez.

en general se trata de personas de escasos recursos con problemas de deserción escolar y carencia de empleo. ${ }^{3}$ Un ejemplo es el municipio de Jiutepec, Morelos. El 27 de enero de 2orI un varón de I/4 años acusado de homicidio que trabajaba para el Cártel del Pacífico Sur mencionó que sólo había estudiado hasta quinto año de primaria y que se encontraba asesinando bajo amenaza de muerte. ${ }^{4}$ De manera similar a lo acaecido en otros sitios, recientemente en Zacatecas la Procuraduría General de Justicia del Estado (PGJE) ha señalado el incremento en la captura de adolescentes narcomenudistas, informantes de grupos delictivos y asesinos. Entre los múltiples casos destaca el de Jorge Armando Moreno Leos, un menor de i3 años sin educación primaria que luego de haber formado parte de los zetas fue ejecutado por dicho grupo en febrero de 2013.5

Durante las últimas décadas, los gobiernos federales y estatales se han mostrado incapaces de brindar adecuados estándares de seguridad a los ciudadanos, aunado a que desde hace algunos años se acentuó la desconfianza sobre las autoridades por la población mexicana, pues en diversas ocasiones los responsables de combatir el narcotráfico mantienen estrechos vínculos con los cárteles y sicarios. ${ }^{6}$ Un ejemplo lo encontramos en el caso del general Jesús Gutiérrez Rebollo, arrestado por haber otorgado protección al narcotraficante Armando Carrillo y participado en el crimen organizado con el cártel de Juárez, siendo el general Gutiérrez comisionado del Instituto Nacional para el Combate a las Drogas durante el gobierno de Ernesto Zedillo (I994-2000).7 De manera similar, el ir de junio de 2005 tuvo lugar en Nuevo Laredo, Tamaulipas, el despido de zoo policías locales luego de que fueran encontradas 44 personas víctimas de secuestro, retenidas ilegalmente por las autoridades municipales. ${ }^{8}$

A semejanza de los casos expuestos, entre $2007 \mathrm{y}$ 2008, Zacatecas también figuró entre las principales entidades del país con militares ligados al crimen organizado, siendo los zetas, el cártel del Golfo y el Cártel de Jalisco algunos de los grupos delictivos con mayor presencia en el estado durante los últimos años. ${ }^{9}$

Cabe señalar que de 2006 a 20ı0, Zacatecas se ubicó entre los principales ocho estados de México donde los cárteles no sólo controlaban parte de su territorio para la distribución de droga sino que también manejaban en gran medida actividades como el comercio informal, el secuestro, la prostitución y la venta de protección. ${ }^{\circ}$

Es posible percibir no sólo el porqué de la desconfianza en las instituciones gubernamentales, sino también el grado de infiltración del narcotráfico en la sociedad y en las instancias encargadas de combatirlo, ya que tanto funcionarios públicos como policías y militares se han incorporado entre los principales actores del crimen organizado, lo cual nos expresa una crisis del Estado mexicano que también se ha hecho presente a largo y ancho de nuestra entidad federativa."

Otras de las causas que han dado pauta al agravamiento de los delitos contra la salud y la vida es una problemática de orden financiero. A inicios del sexenio presidencial 2006-20I2 casi todos los recursos asignados a las fuerzas armadas en México se destinaban al pago de personal, lo cual propició el atraso en 

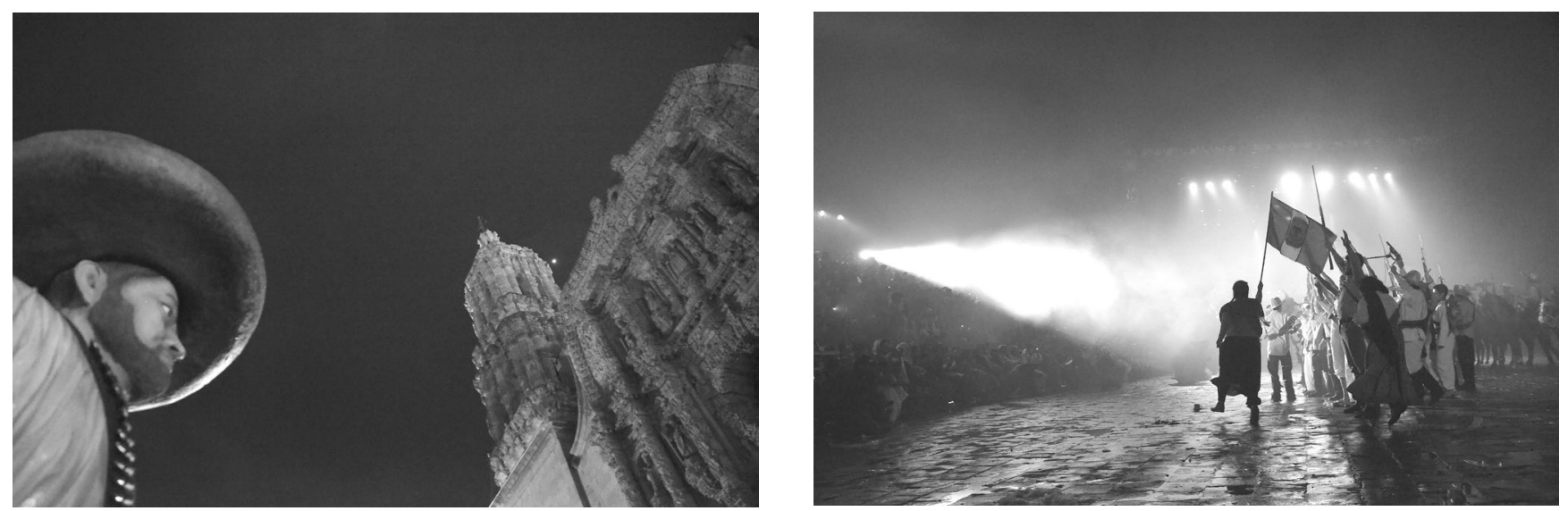

La celebración del centenario de la Toma de Zacatecas, una gesta popular revolucionaria, tuvo lugar en momentos en que se adoptan reformas constitucionales de talante contrarrevolucionario.

Foto: Jorge Vázquez.

la adquisición de armamentos eficaces y mejores equipos para los militares. ${ }^{22}$

En cuanto a Zacatecas, la fuga de 53 reos zetas del penal de Cieneguillas, suscitada el i6 de mayo de 2009, representaba un ejemplo no sólo de la corrupción de los 40 custodios, comandantes y director de la prisión, sino que también una prueba de la incapacidad del gobierno y sus estructuras de seguridad para hacer frente al crimen organizado. Tal suceso implicó complicidad tanto en el penal como en altas esferas gubernamentales del estado. ${ }^{13}$

De igual manera, la adquisición de armas de alto calibre y artillería pesada por los narcotraficantes ha dificultado el enfrentamiento entre dichas agrupaciones y las defensas nacionales y estatales, aunado a que los diferentes cárteles también han comenzado a crear sus propias unidades de inteligencia, al parecer principalmente con la ayuda de kaibiles (soldados que pertenecían a las fuerzas especiales guatemaltecas) y ex integrantes del Ejército mexicano. ${ }^{14}$

Dichas agrupaciones, además del adiestramiento, han conformado verdaderos ejércitos; además de los kaibiles, han atraído sicarios de otras naciones y antiguos miembros de los grupos aeromóvil y anfibio de las fuerzas especiales que fueron entrenados por la estadounidense Agencia Central de Inteligencia (CIA, por sus siglas en inglés)..$^{5}$

LEgALIZACIÓN DE LAS DROGAS: OPINIONES CONFRONTADAS

Desde hace años, se han discutido posibles soluciones al narcotráfico y a la muerte de inocentes causadas por la guerra contra las drogas. Entre las alternativas se ha propuesto la legalización de las drogas, al considerar que su decreto podría finiquitar la guerra y el asunto se reduciría a la voluntad y libre decisión de los individuos por ingerir o no las drogas. ${ }^{6}$

De acuerdo con la visión señalada, si el Estado regulase la venta de los narcóticos, únicamente consumirían esta clase de sustancias quienes gustasen de hacerlo sin necesidad de adquirirlas clandestinamente. El 2I de octubre de 20Io, el diputado Víctor Hugo Cirigo presentó una iniciativa de ley donde solicitaba legalizar el consumo de drogas en el país, específicamente la marihuana. ${ }^{7}$

En Zacatecas, en agosto de 2013, el diputado José Olvera Acevedo consideró viable la legalización de las drogas y permisible que se considere una cierta cantidad de gramos para cada droga, para lo cual se analizaría quién se haría cargo de la comercialización de dichas sustancias. ${ }^{18}$

No obstante la viabilidad de las propuestas señaladas, el narcotráfico no sólo se trata de un dilema comercial, sino que constituye una problemática de violencia, secuestro, delitos contra la salud, compra de voluntades y otras clases de actividades delictivas. ${ }^{19}$

Por los motivos señalados, tanto el presidente Enrique Peña Nieto, como el ex presidente Felipe Calderón se pronunciaron en contra de la legalización de las drogas. Calderón señaló que para erradicar la violencia es necesario reconstruir el tejido social con oportunidades de educación, trabajo y salud, ya que con la legalización de la droga sólo se incrementaría su consumo, y se generaría un incentivo económico para la operación delincuencial. ${ }^{20}$

De manera similar, el gobierno de Zacatecas (201020I6) ha manifestado una incipiente preocupación por la problemática de las drogas. La prevención de las adic- 

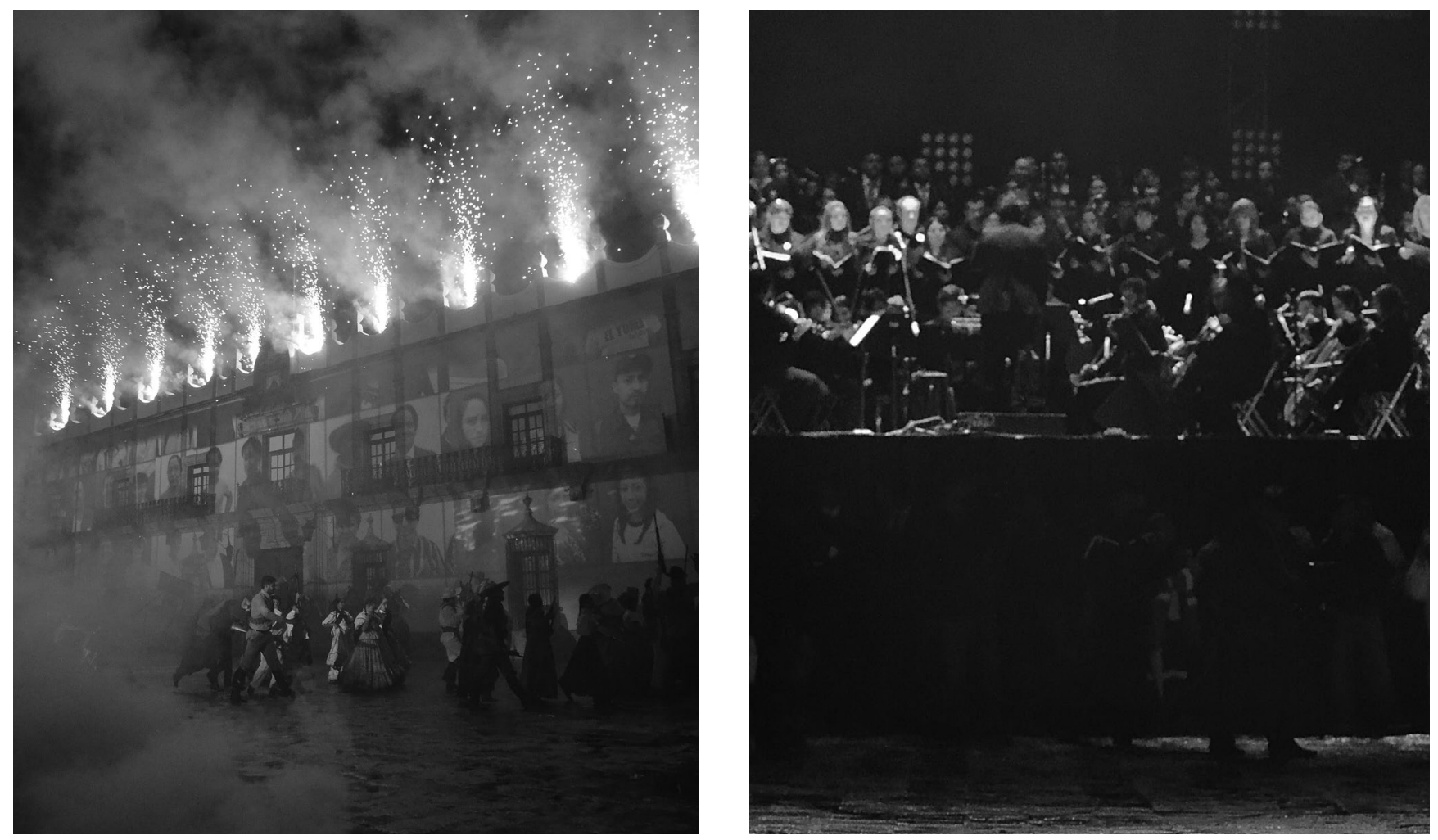

La promoción de espectáculos masivos ha sido la tónica del gobierno para entretener a la población con un derroche de recursos que contrasta con la situación de pobreza, precariedad laboral e inseguridad humana en Zacatecas. Foto: Jorge Vízquez.

ciones es uno de sus intereses, pues además de la imConclusión plementación del Programa de Prevención del Delito en Zacatecas y la Guía de Orientación para la Prevención de Adicciones en Preescolar presentados en 2013, desde 2012 fue puesta en marcha la estrategia Alianza de Jóvenes Contra las Adicciones y la Violencia en el Estado, la cual ha sido coordinada con asociaciones civiles como los Centros de Integración Juvenil. ${ }^{21}$

No obstante lo realizado, hasta ahora han sido pocos los avances materializados en el combate al uso de drogas. Una prueba es el incremento del consumo de marihuana registrado en la entidad de 8.8 por ciento durante 2013, además del consumo de crack por encima de la media nacional. ${ }^{22}$

Según la Encuesta Nacional de Victimización y Percepción sobre Seguridad Pública realizada por el Instituto de Estadística, Geografía e Informática (INEGI) en 20I3, la tasa de delitos en Zacatecas por cada ıoo,ooo habitantes ascendió de I8,772 en 20I a 20,5o6 durante 20I2, siendo uno de los diez estados con mayores defunciones por homicidio. Además, Zacatecas figura entre las entidades con mayor atención del gobierno federal para la estrategia antisecuestros, pues desde 2013 la entidad se incluye entre los diez estados con mayores índices de secuestro a nivel nacional. ${ }^{23}$

La violencia, el crimen organizado y la inseguridad en el estado de Zacatecas continúan figurando entre las problemáticas de mayor interés tanto para la población zacatecana como para el gobierno federal y estatal.

No obstante, es necesario revisar si las estrategias para reducir y prevenir las problemáticas de seguridad en el ámbito local-regional son adecuadas, ya que además de la evidencia de éxito y de la evaluación del impacto alcanzado por los programas, las características históricas, territoriales, gubernamentales, institucionales y del entorno, deberán ser consideradas como base en la construcción político-social de planeaciones eficaces para el combate a la delincuencia en el estado.

Finalmente, para impedir un estancamiento o retroceso a los estándares de seguridad hasta ahora alcanzados, es indispensable analizar que las políticas implementadas en la entidad no correspondan a una conveniencia política del gobierno en turno, sino que realmente constituyan estrategias de seguridad sostenibles a largo y mediano plazo, con incidencia en el mejoramiento tanto de las condiciones de bienestar poblacional como de las estructuras de seguridad y unidades para la atención de la violencia. 


\section{- notas $\cdot-$}

'Gobierno del Estado de Zacatecas (2003), "Ley para prevenir y atender la violencia familiar", Periódico Oficial del Estado de Zacatecas, i9 de febrero. <http://www.ife.org.mx/docs/IFE-v2/ DECEYEC/DECEYEC-IFEMujeres/Mujeres-Legislacion/Mujeres-LegislacionLocal-estaticos/Zac_Ley_prevencion_violencia.pdf $>$. Gobierno del Estado de Zacatecas (2009), "Ley de acceso de las mujeres a una vida libre de violencia", Periódico Oficial del Estado de Zacatecas, I7 de enero.http://www.tsjzac.gob.mx/documentos/leyes_tratados/Leyes_Estado/ıo.pdf.

"Raúl Benítez (2009), "La crisis de seguridad en México", Nueva Sociedad, núm. 220, pp. 176-г78. <http://www.nuso.org/ upload/articulos/36or_r.pdf; Paris Martínez (2012), "Esclavos del narco: Profesionistas forzados", Animal Político, 3o de octubre $<$ http://www.animalpolitico.com/2012/Io/esclavos-del-narcolos-esclavos-especializados/\#axzzexfFtAjM5 > S Sandra Marina (2014), "Han muerto 400 soldados en la lucha contra el narcotráfico", i3 de enero <http://www.elfinanciero.com.mx/sociedad/ han-muerto-40o-soldados-en.html .

${ }^{3}$ Norberto Emmerich (20II), "Cruce de fuego: niños, niñas y adolescentes en el narcotráfico mexicano", Documentos de Trabajo, núm. 274, p. I9. ‘http://www.ub.edu.ar/investigaciones/dt_nuevos/274_Emmerich.pdf ; Jorge Mercado (2006), "Violencia y migración, una representación colectiva. Estudio de caso en el municipio de Fresnillo", Zacatecas, Sociológica, núm. 62, pp. ı75I96. $\langle$ http://www.revistasociologica.com.mx/pdf/6208.pdf .

4Emmerich (20II), op. cit., pp. I9-20.

${ }^{5}$ Cristo González (2014), "Deserción escolar 'alimenta' al crimen organizado", NTR, I3 de enero.http://ntrzacatecas. com/20I//oI/I3/desercion-escolar-alimenta-al-crimen-organizado/; Magda Coss (2013), "Dos historias de abandono y violencia", MDZ, 4 de Abril. <http://www.mdzol.com/nota/457303/>.

${ }^{6}$ Benítez (2009), op. cit, p. I86.

¿Enrique Krauze (2010), "México contemporáneo (19882008)", en Gisela Von Wobeser (coord.), Historia de México, México, FCE, p. 274 .

${ }^{8}$ Benítez (2009), op. cit., p. 186.

${ }^{9}$ José Luis Cisneros (2010), "El cáncer del narcotráfico y la militarización de la seguridad pública", El Cotidiano, núm. ı6ı, p. 5ı. <http:/www.redalyc.org/articulo.oa?id=325ı3865oo7>; José Antonio O' Quinn y Roberto Reyes (2013), "La comunicación gubernamental de la guerra contra el narcotráfico en México", Espacios Públicos, núm. 36, p.59. ‘http://www.redalyc.org/articulo. oa?id=676269130o $>$; Carlos Rentería (2013), "Hombres armados controlan Zacatecas", El Sol de Nayarit, I de diciembre. ‘http:/ www.elsoldenayarit.mx/nota-roja/2369 I-hombres-armados-controlan-zacatecas $>$.

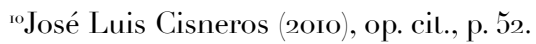

"Krauze (2010), op. cit., p. 274.

"Benítez (2009), op. cit., pp. I85-186.

${ }^{13}$ Georgina Saldierna, Alfredo Valadez y Gustavo Castillo (2009), "Comando de 8 o zetas saca de un penal de Zacatecas a 53 presos", La Jornada, I7 de mayo. <http://www.jornada.unam. $\mathrm{mx} / 2009 / 05 / 17 /$ politica/oo3nipol $>$.

«Benítez (2009), op. cit., pp. I85-ı86; Víctor Solís (20II), “De dónde vienen las armas del narco?", El Universal, II de febrero. http://www.eluniversal.com.mx/notas/744098.html .

${ }^{15}$ Krauze (2010), op. cit., p. 274.

${ }^{16} J u l i a ́ n$ Germán Molina (2013), "La legalización de las drogas en México", Milenio, 8 de noviembre. <http://www.milenio.com/ firmas/julian_german_molina_carrillo/legalizacion-drogas-Mexico_I8_I86-61/403.htmb.

${ }^{17}$ Molina (2013), op. cit.

${ }^{18}$ Alma Alejandra Tapia (2013), "Tienen diputados locales posturas encontradas en el tema de las drogas", La Jornada, 12 de agosto. http://issuu.com/lajornadazacatecas.com.mx/docs/local_12082013>.

19Zaira Ramírez (2014), "Legalizar mariguana no resuelve problema de fondo: Calderón”, Informador, 7 de marzo. 〈http:// www.informador.com.mx/mexico/20ı//5ı68ı5/6/legalizar-mariguana-no-resuelve-problema-de-fondo-calderon.html .

${ }^{20}$ Ramírez (2014), op. cit.; Molina (2013), op. cit.

"Alma Ríos (2012), "Unen esfuerzos para prevenir adicciones", 24 de Mayo <http:/anuario.ajusco.upn.mx/2or2/index.php/ imagen/6o778-unen-esfuerzos-para-prevenir-adicciones-.html $>$; Thelma Lorena Rossoy Fuensanta Casanova (2013), "Guía de orientación para la prevención de adicciones en escuelas de educación preescolar", Guadalupe, Gobierno del Estado de Zacatecas. http://www.seduzac.gob.mx/portal/documentos/Guia_Escuela_ Segura.pdf ; Alfredo Valadez (2013), "Arranca programa de prevención de delito en Zacatecas", La Jornada, 3o de octubre. ‘http:// www.jornada.unam.mx/20I3/1o/3o/estados/o3/nn2est .

${ }^{22}$ Antonio Torres (2014), "Incrementó 8.8 por ciento consumo de marihuana en 2013: CIJ", NTR, 29 de enero, <http:/ntrzacatecas. com/2OI//or/29/incremento-8-8-consumo-de-marihuana-en2013-cij/>.

${ }^{23}$ Instituto Nacional de Estadística y Geografía (INEGI) (2013), $<$ Encuesta Nacional de Victimización y Percepción sobre Seguridad Pública 2013 (ENVIPE) $\gg$, Boletín de Prensa, número 39o, pp. 7-I/. <http:/www.inegi.org.mx/inegi/contenidos/espanol/prensa/ boletines/boletin/comunicados/especiales/2013/septiembre/comunicar5.pdf $>$; Lizbeth Padilla (2014), $<$ México combatirá el secuestro con inteligencia y evaluación de unidades $>>, \mathrm{CNN}, 28$ de enero. $<$ http://mexico.cnn.com/nacional/20I//oI/28/estrategia-antisecuestro-mexico-seguridad-pena-nieto-segob> 


\section{SEPTIEMBRE}

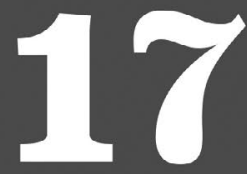

2014

10 A 15 HRS

SEMINARIO

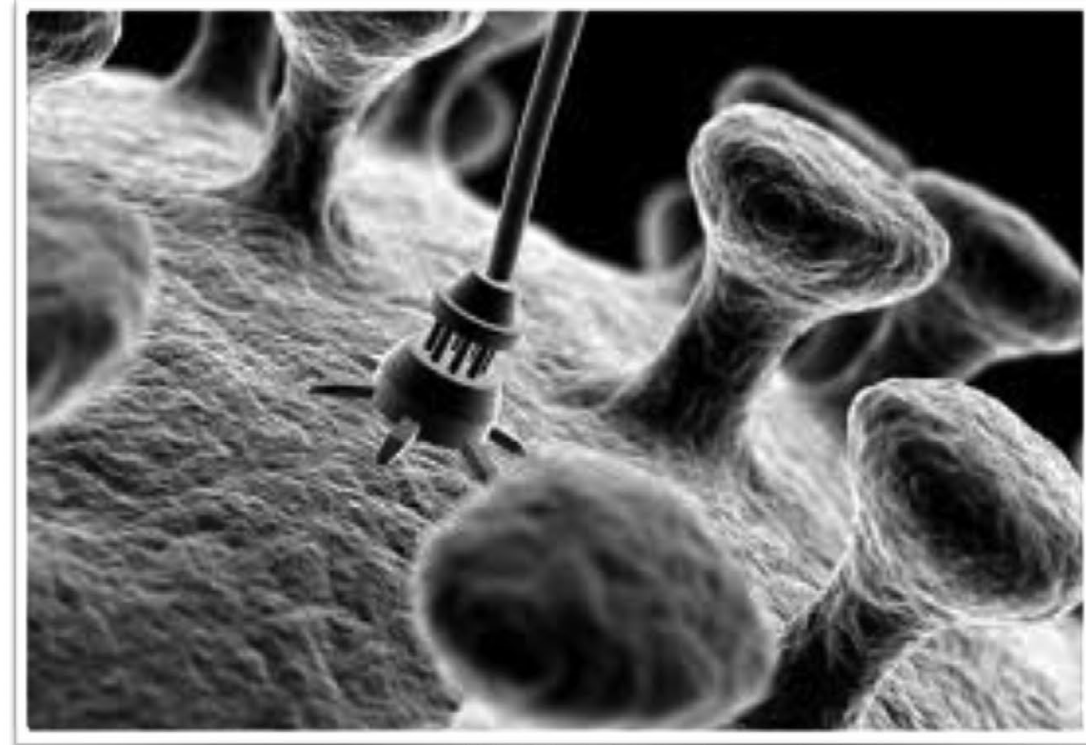

NANOTECNOLOGÍA \& SALUD: NUEVOS RETOS

$$
\stackrel{\star \star}{\star \star \star \star \star ~}_{\star \star}^{\star}
$$

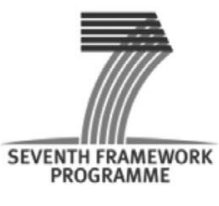

PRTH FRAMEWO
PROGAMME
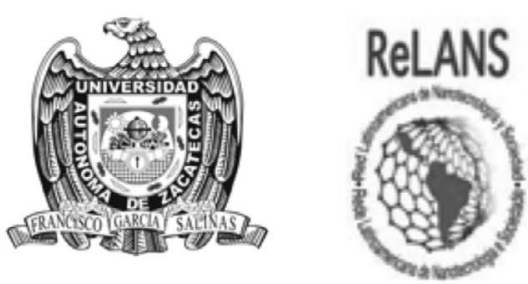

ESTUDIOS DEL DESARROLLO

Auditorio de la Unidad Académica en Fistudios del Desarrollo, UAZ. Participan:

Dndo. David Fajardo-Ortíz (Fac. Medicina, UNAM)

Dr. Eduardo Robles-Belmont (IIMAS, UNAM)

Dr. Alejandro Aguilera Galaviz (IyP, UAZ)

Dr. Pascual García Zamora (Maestría Ciencias de la Salud, UAZ)

Dndo. Miguel García Guerrero (Unidad Académica en Estudios del Desarrollo, UAZ)

Dnda. Laura Vidal Correa (Unidad Académica en Estudios del Desarrollo, UAZ)

Dr. Guillermo Foladori (Unidad Académica en Estudios del Desarrollo, UAZ)

Dr. Edgar Záyago Lau (Unidad Académica en Estudios del Desarrollo, UAZ)

Se entregarán certificados de asistencia 